\title{
Current research activities on holographic video displays
}

Levent Onural

Fahri Yaras

Hoonjong Kang 


\title{
Current research activities on holographic video displays
}

\author{
Levent Onural, Fahri Yaraş and Hoonjong Kang \\ Dept. of Electrical and Electronics Eng., Bilkent University, Ankara, Turkey
}

\begin{abstract}
"True 3D" display technologies target replication of physical volume light distributions. Holography is a promising true 3D technique. Widespread utilization of holographic 3D video displays is hindered by current technological limits; research activities are targeted to overcome such difficulties. Rising interest in 3D video in general, and current developments in holographic 3D video and underlying technologies increase the momentum of research activities in this field. Prototypes and recent satisfactory laboratory results indicate that holographic displays are strong candidates for future 3D displays.
\end{abstract}

Keywords: holographic video, electro-holography, digital holography, spatial light modulators, 3DTV

\section{INTRODUCTION}

True 3D displays have always been the ultimate goal in visual communications. ${ }^{1}$ What discriminates a "true 3D" display from the others is the targeted goal: True 3D techniques attempt to physically duplicate the light that fills a 3D volume. In other words, true 3D techniques do not rely on human perception to yield a sense of 3D. If the duplication of the volume filling light field is achieved perfectly, then the observer of the duplicated light, whether it is a camera, an animal or a human, will capture, or perceive, the same visual content as if looking at the original. Assuming that the original was a $3 \mathrm{D}$ scene with $3 \mathrm{D}$ objects in it, the observer who is looking at the duplicate, which may be created at a different time, at a different place, will see the same 3D environment. Original and its duplicate will be optically indistinguishable.

Currently available 3D displays, on the other hand, are usually stereoscopic, and therefore rely on human perception for 3D observation. Stereoscopic technology is almost 170 years old. ${ }^{2}$ Since the target in stereoscopy is only to replicate the two focused retina images, one for each eye, the technique is far from a true $3 \mathrm{D}$ display. ${ }^{3}$ The human visual system will not receive an identical optical stimulus compared to the original input, when watching stereoscopic video, and therefore, the quality of perception is inferior. The unnatural features of the stereoscopic stimulus results in a viewing discomfort which is similar to motion sickness. ${ }^{4,5}$ Even though current end-to-end digital techniques, and restricted parameters reduced this problem significantly, it is always there, at different degrees for each observer, due to intrinsic problems associated with the stereoscopy.

The two techniques which target true $3 \mathrm{D}$ viewing are the integral imaging and holography. ${ }^{6-10}$ Integral imaging attempts to recreate the original optical light field distribution using lens arrays ${ }^{11}$ and operates also with incoherent light; incoherent light is a desirable feature in displays. Analysis of integral imaging systems are usually based on ray optics techniques. Holography, on the other hand, attempts to recreate wavefronts based on principles of diffraction and interference. Coherent light is a must in capturing holograms, but this may be relaxed during the display phase.

Here in this paper, an overview of current state-of-the art in holographic display research is presented. Some prototypes from different laboratories are mentioned together with few commercial prototypes. Recent results from research at Bilkent University is presented.

Some of the problems in holographic displays are due to parameters of underlying electro-optical components that are available today; such limitations prevent easy solutions for satisfactory displays. ${ }^{12,13}$ Proposed methods to somewhat relieve such restrictions include head-tracking systems which render holograms for small angle reconstructions that only accommodate the two tracked pupils of the observer; the hologram is refreshed as the observer moves. ${ }^{14}$ Other group of currently studied systems include multi-SLM based solutions; ${ }^{15}$ time multiplexing is also utilized. ${ }^{16}$

\footnotetext{
Send correspondence to L. Onural onural@bilkent.edu.tr
} 
Another group of problems arise during the generation of holographic signals which are to be written on the display device. There are two common approaches: holographic signals are either captured by holographic camera set-ups, ${ }^{17,18}$ or those signals are computationally generated from a given 3D input scene which is described using computer graphics techniques. ${ }^{19}$ In either case, significant processing is needed. In the first group, the captured holographic signals must be converted to interface the capture-end and display-end properties and parameters; for the second group, the signals must be custom-generated to fit the display-end characteristics. Real-time processing to support video is quite difficult since the processing requirements are demanding. ${ }^{20,21}$ Certain approximations, which in turn show up as degradations, are usually adopted. Furthermore, certain techniques which employ suitable fast computer architectures, like GPU based processing, or special purpose programmable hardware structures are used. ${ }^{15,22-30}$

In Section 2 an overview of various holographic display designs from different laboratories are presented. In Section 3 a real-time phase-only holographic video display system from Bilkent University is outlined. In Section 4 a GPU-based fast algorithm for real-time holographic frame generation algorithm at video rates from Bilkent University is outlined. In Section 5 a brief outline of a multi-SLM design specifications are indicated. Conclusions are drawn in Section 6.

\section{OVERVIEW OF CURRENT STATE-OF-THE ART IN HOLOGRAPHIC DISPLAY RESEARCH}

Many prototypes of dynamic holographic displays and related experiments have been reported in the literature. Usually liquid crystal devices are used. Michalkiewicz et al. reported electro-holographic reconstructions by LCoS devices. ${ }^{31}$ Holographic video displays using LCoS spatial light modulators together with a light-emitting diodes (LEDs) are discussed by Ito et al. and electro-holographic reconstructions are reported. ${ }^{32}$ Holographic display systems with an LCoS technology and LED illumination are also discussed by Yaras et al. ${ }^{33,34}$ In their work, inline phase holograms are calculated by iterative algorithms and phase-only spatial light modulators are used for holographic reconstructions. Time and space multiplexing of SLMs is one of the common approaches. Sato et al. use time division switching of lasers by using electronic shutter to obtain color holographic reconstruction. ${ }^{35-37}$ In addition, Shimobaba et al. proposed a method that uses time multiplexing of LEDs for color holography. ${ }^{16,38}$ Another color holographic display system was discussed by Ito et al. ${ }^{39}$ Different from previous methods, they use space multiplexing to generate color holographic reconstructions. In recent papers, Ohmura et al. reported a method to increase the viewing angle of the hologram by using a mirror module. Another color holographic display system was proposed by Takano et al.: ${ }^{40-43}$ they used a metal halide lamp to illuminate the SLMs. In other papers from the same group, a full-color electro-holographic 3D display system with LED illumination is reported. ${ }^{44,45}$ Hahn et al. proposed a holographic display system with a curved array of SLMs. ${ }^{46}$ They used the the holographic stereogram technique for generating holograms. The viewing angle is larger due to the curved structure.

An early prototype is developed by the Spatial Imaging Group at MIT Media Lab; ${ }^{47-54}$ the holographic display systems from MIT (Mark-I, Mark-II and Mark-III) use acousto-optic modulators or guided-wave optical scanners to render volumetric objects in video frame rates. A commercial holographic display system prototype is produced by SeeReal. ${ }^{14,55-58}$ The distinguishing feature of their approach is the reconstruction of only the portion of the wavefront originating from the object that actually enters the eye pupils of an observer. Yet another company, QinetiQ, developed the so called Active Tiling system. ${ }^{59}$ The system uses a high frame rate electrically addressed SLM to tile the hologram onto a non-pixellated optically addressed SLM. The system is able to display more than 100 megapixels digital holograms. A research group in Chiba University developed a holographic display system, named Horn (HOlographic ReconstructioN). ${ }^{26,60-65}$ In their systems the computer generated holograms are calculated by using field programmable gate arrays (FPGAs). The latest version, so called Horn6 , has four FPGA chips and uses a look-up table to calculate digital holograms at video frame rates. Another electro-holographic system is developed by National Institute of Information and Communications Technologies (NICT). ${ }^{66}$ The system has three major parts: a capturing system, a computing system and a holographic display system. The capturing system has a ultra-high-definition $(8 K \times 4 K)$ integral imaging camera. Digital hologram of the captured scene is calculated in real time and displayed by using three SLMs. 


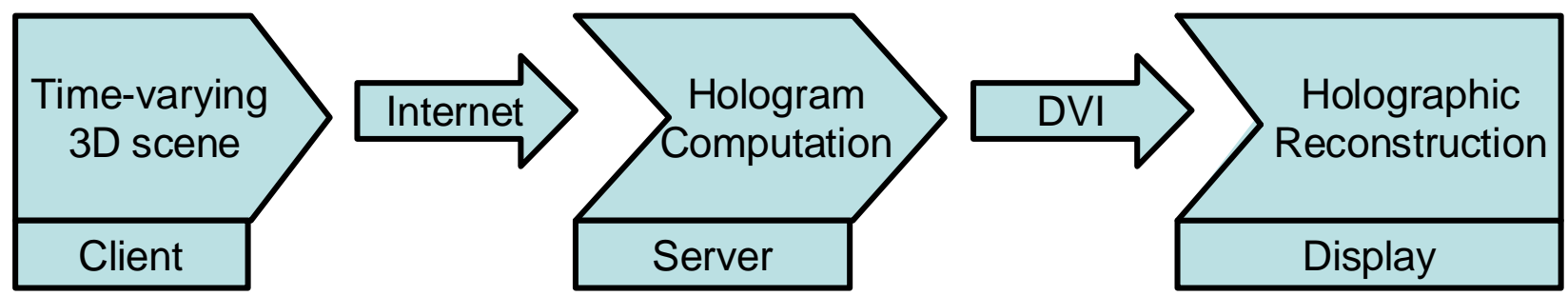

Figure 1. End-to-end system

\section{REAL-TIME PHASE-ONLY COLOR HOLOGRAPHIC VIDEO DISPLAY SYSTEM}

The proposed system consists of three major components (See Fig. 1). ${ }^{15,25}$ The first component is the client where 3D model of the object is stored. The second part is the server. The 3D information that is stored in the client is sent to the server through an internet connection. The server calculates the color hologram in real-time and directs it to the display which is the third component of the overall system. The display part consists of spatial light modulators (SLMs), optics and light sources. Reconstructions were recorded with the help of a CCD camera without a lens on it.

\subsection{Algorithm}

Fast computation of the digital holograms is essential in real-time holographic displays. In our system we use Accurate Compensated Phase-Added Stereogram (ACPAS) ${ }^{22}$ which is proposed by Kang. It is an enhanced version of Compensated Phase-Added Stereogram (CPAS). ${ }^{23}$ CPAS algorithm is slightly faster; however, ACPAS gives better results in terms of reconstruction quality. The reconstructions from holograms that are generated by ACPAS are similar in quality to those from Fresnel holograms. Moreover, computation time of ACPAS is shorter than the Fresnel holograms due to employed approximations and the nature of the algorithm which can be implemented in parallel. Therefore it is suitable for real-time applications. The details of the algorithm is presented in Section 4

\subsection{Client and Server}

Time-varying scene of our rigid 3D model is stored in the client. The model consists of discrete points floating in the space (point-cloud model). For each frame of the 3D scene, 3D information for each object point (3D coordinates and the color) are sent to the server through the internet. When the server receives the $3 \mathrm{D}$ information, it immediately starts to compute the hologram of that $3 \mathrm{D}$ scene. The hologram is calculated by the ACPAS algorithm. ACPAS first starts with an empty hologram and divides it into square size segments. Each object point in the point cloud has a contribution within a segment. The contribution from a single point onto a segment is approximated as a single complex sinusoid weighted by a corresponding amplitude. Finally, within a segment, all contributions from object points are superposed. The details of the algorithm is presented in Section 4.

Holograms are calculated by using graphical processing units (GPUs). Segmented structures of the holograms are suitable for parallel processing; therefore, GPUs speed up the computation. Furthermore, in order to increase the frame rate, three GPUs are used and pipelined to achieve real-time computation. Since our spatial light modulators are phase-only, we discard the amplitude of the resultant complex fringe pattern and we only use the phase information as a phase hologram. This process is executed for each color (red, green and blue) and the resultant phase holograms are merged into a single RGB bitmap image. The computer graphics unit sends that RGB image to the driver of the spatial light modulators.

\subsection{Display}

The display part consists of light emitting diodes (LEDs), spatial filters, beam expanders, non-polarized beam splitters and SLMs (See Fig. 2). The light that emerges from LED first passes through a spatial filter. This increases the spatial coherence of the light. Then beam expander collimates the light to obtain a plane wave. Each 


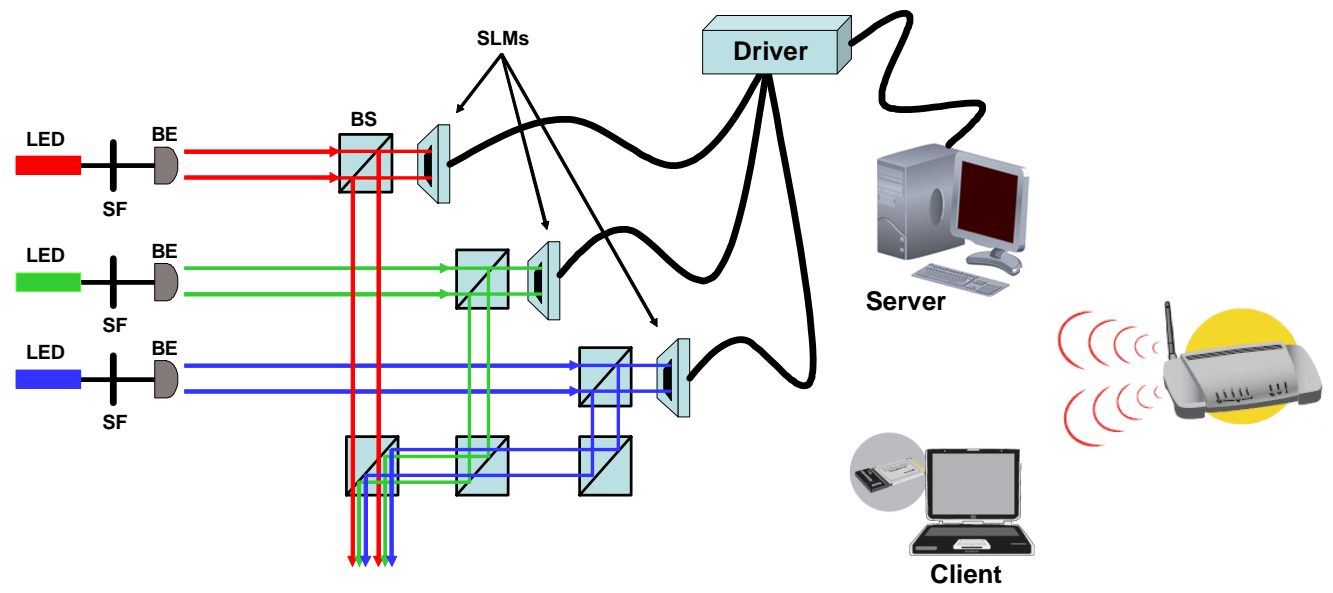

Figure 2. Overall setup: BE, beam expander; SF, spatial filter; BS, beam splitter

color beam hits the corresponding SLM and gets modulated by the phase hologram loaded onto the SLM. The modulated light reflects back and with the help of beam splitters/combiners all individual color reconstructions are overlapped. Reconstructions can be recorded by a camera or can be observed by naked eye.

\subsection{Results}

It is observed that scenes that contain 10,000 object points can be displayed in real-time by using three GPUs. The proposed system also shows that the point-based 3D representation can be transmitted through the network for real-time applications. Although LEDs have a wider spectrum, reconstructions are satisfactory. The viewing angle is quite limited due to pixel size of the SLMs; it is possible to view the reconstructions within an angle of only few degrees. Furthermore, the reconstruction size is in the order of the size of the SLMs (order of a cm.). As a result, it can be concluded that the proposed system can be used as a color holographic video display system within the limitations as mentioned above.

\section{FAST DIGITAL HOLOGRAM GENERATION ALGORITHM}

The accurate compensated phase-added stereogram (ACPAS) is one of the candidates in the reported methods so far. ${ }^{20,27,67-70}$ The quality of reconstruction is similar to the quality obtained from direct hologram generation algorithms; furthermore, the algorithm can be executed quite fast using parallel architectures. ${ }^{15,24,25,71-74}$ The ACPAS has following characteristics: partitioning the hologram plane, significantly reduced computational complexity, parallelizable algorithm, and computation of each segment using inverse fast Fourier transform (IFFT). As a first step for the computation of the ACPAS, a hologram plane is partitioned into square segments. The contribution from each object point to associated segment is determined for each segment. This process is repeated for all object points, and these contributions are superposed on the associated segments. Finally, each segment is transformed by IFFT. A perspective image of the used 3D model and numerical reconstructions from the Rayleigh-Sommerfeld (R-S) integral and the ACPAS are shown in Fig. 3. As seen Fig. 3, the qualities of the reconstructed images from the generated fringe pattern by using the ACPAS and the R-S are similar.

In case of the direct methods such as computation of Rayleigh-Sommerfeld integral or its approximations, the contribution of each object point corresponding to each pixel on the hologram plane is calculated, and thus, the computational complexity of such a method is $O(n m)$, where $n$ is the number of pixels of the digital hologram and $m$ is the number of object points. ACPAS is based on partitioning the hologram plane, and the contribution of each object point is calculated for each segment. Therefore, the computational complexity is significantly reduced to $O(m s)$, where $s$ is the number of segments. In addition, the computation of the contribution from each object point to a segment and the IFFT process per segment are standalone processes. Therefore, ACPAS can be accelerated on parallel computing platforms. In our system, the ACPAS is implemented on two different computing platforms: a graphic processing unit (GPU) based platform and a central processing unit (CPU) 


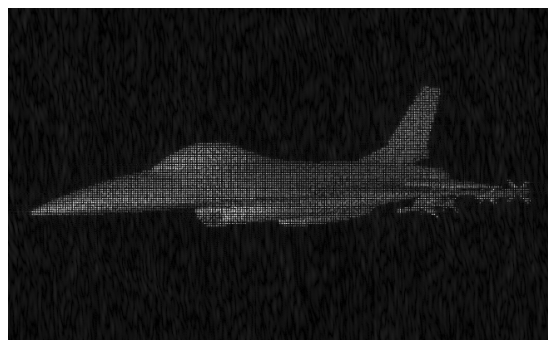

(a) 3D model

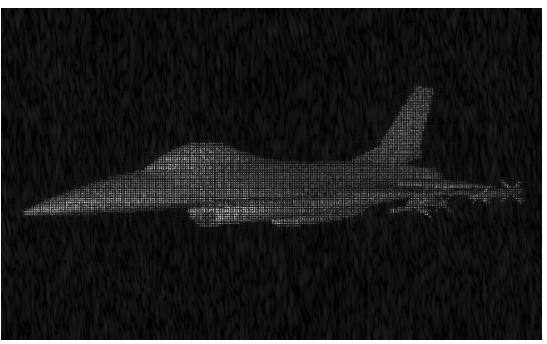

(b) Rayleigh-Sommerfeld integral

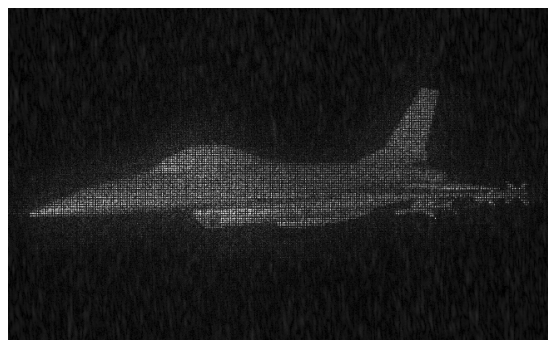

(c) ACPAS

Figure 3. A perspective image of the used 3D model and reconstructed images.

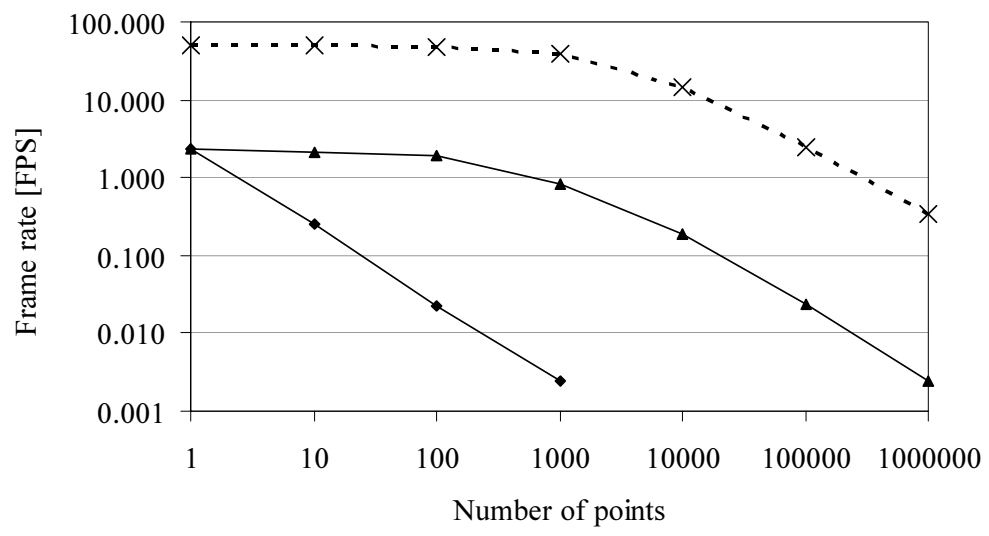

$\longrightarrow$ Rayleigh-Sommerfeld on CPU $\longleftarrow$ ACPAS on CPU

- - - - ACPAS on GPU

Figure 4. Computational performances of the algorithms for different number of object points.

based platform, and the computation times are measured. The results are shown in Fig. 4. The computation time of direct implementation of the R-S integral is used as a reference. As shown in Fig. 4, the ACPAS on the GPU-based platform is significantly accelerated (up to 300 times faster than the ACPAS on CPU-based platform, and up to 130 thousand times faster than the reference on CPU-based platform for one million object points) and video frame rate is achieved for 10,000 object points. The used parameters are as follows: the hologram size is $1 K \times 1 K$ pixels, the segment size is $32 \times 32$ pixels, and the IFFT size is $64 \times 64$ pixels. The computing system has an Intel(R) Xeon(R) CPU 2GHz, 8-GByte memory and a NVIDIA $®$ Geforce $($ GTX 285 video cards. The ACPAS algorithm is implemented by using NVIDIA's compute unified device architecture (CUDA $)^{\mathrm{TM}}$ technology

The GPU provides single and double precision arithmetic for floating point computation. The computational speed of the single precision arithmetic on GPU is much faster than the double precision case, however the accuracy of the single precision is degraded due to the restricted available length of floating point data. ACPAS uses phase-compensation method to improve the quality of the reconstruction, thus, the ACPAS on the GPU with single precision results in significant phase errors because the phase computation is sensitive. The accuracy of the ACPAS algorithm for single and double precision arithmetic is evaluated. ${ }^{24}$ Peak signal-to-noise ratio (PSNR) is used as a measurement method for the numerical reconstructions from the fringe patterns by using the ACPAS in two different floating point precision. Then the reconstruction from the R-S hologram was used as the reference. The PSNR levels are measured according to the increasing number of object points. As a result, the PSNR levels compared with the reference case was almost the same for single and double precision arithmetic computation. Since the available numbers of bits of single and double precision format are different, the phase distributions of fringe patters with single and double precision are also different. However, the reconstruction 

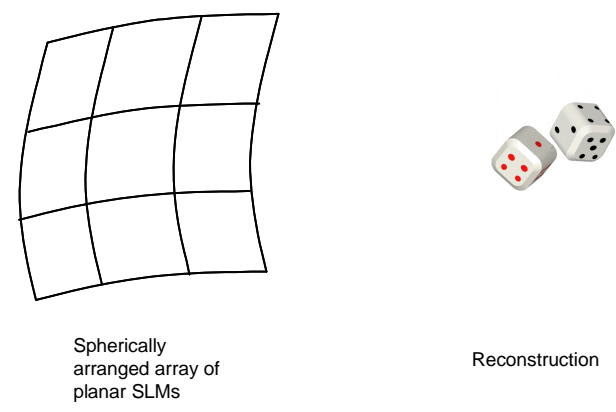

Figure 5. Spherically arranged electro-holographic display design

qualities are comparable. Therefore, the ACPAS can be further accelerated on GPU computing platform with single precision arithmetic without significant quality degradation.

\section{SPECIFICATIONS OF A SATISFACTORY QUALITY HOLOGRAPHIC DISPLAY SYSTEM}

Appropriate space-bandwidth product of the hologram has to be specified for a comfortable viewing angle in electro-holographic displays. The bandwidth of the holograms may be restricted based on the limitations of the human visual system. The maximum spatial frequency of the human visual system determines the maximum bandwidth of the hologram and therefore the maximum diffraction angle. Limitation of the diffraction angle indicates the minimum hologram size for a stationary observer. In addition, if the observer is free to move along the lateral direction, hologram size increases accordingly. If the display system supports also the rotational motion of the human observer, bandwidth and the size of the hologram both increase. Therefore, for a comfortable viewing angle, space-bandwidth product has to be significantly larger than the stationary observer case. Alternatively, large space-bandwidth product may be relieved by implementing an adaptive systems which tracks the observer location and adjusts the illumination angle of the SLM, instead of a stationary orthographic illumination.

If the Nyquist rate is satisfied and low-pass filtering is used to recover the original field, a low-pass filtering prior to sampling is required to avoid aliasing. This is achieved by using a 4-f system with a spatial filter. Furthermore, the pixellated nature of the SLM creates higher diffraction orders during the display. If a low-pass filter is not used right after the SLM, required pixel sizes decrease significantly to eliminate the overlapping of diffraction orders.

In a study, it is shown that higher than 4 -bit quantization gives satisfactory results. ${ }^{75}$ Therefore we can conclude that the number of quantization levels of the available SLMs is more than needed. Speckle noise is another issue in holographic displays. There are many methods to eliminate this undesirable effect. One of the possibilities is to use LEDs. LEDs have both time and space coherence to some extent. Although due to low coherence characteristics reconstructions might be somewhat blurred, there is no observable speckle noise. Reconstructions can also be observed by naked eye when LEDs are used.

Reconstruction quality when the SLM is illuminated by an oblique incident light is examined. Based on our experiments we can conclude that the results are still satisfactory up to 25 degrees of tilting. There are also polarization issues for tilted reconstructions. From the experiments, we again show that reconstructions are still satisfactory up to 20 degrees of tilting and we can use this result for the design of a cylindrical holographic display.

In addition to oblique incident illumination of the SLMs, need for off-axis holograms arises in order to get rid of the undiffracted beam. However, this increases the maximum frequency content of the holographic pattern. In order to support those higher frequencies, pixel periods should be decreased. Therefore we can conclude that using off-axis hologram may change the required pixel size significantly. In addition, since tilting of the reference beam is in one direction, pixel periods only in corresponding direction will decrease; and therefore, non-square pixel shapes will appear. 
Most of the electro-holographic display systems are planar. However, since planar holographic displays require more space-bandwidth product to satisfy motion and rotations, necessity of using circular or spherical configuration of discrete SLM patches is obvious. Fig. 5 shows a spherically arranged electro-holographic display design.

\section{CONCLUSIONS}

Holographic displays are good candidates for futuristic true-3D displays. Research activities on holographic displays are gaining momentum throughout the world, and promising preliminary results have been demonstrated. There are even some commercial prototypes. As the underlying technologies further develop, and as better holographic display designs appear, satisfactory quality holographic displays with fewer constraints may emerge. Computational performance associated with generation and processing of holographic signals will certainly improve as more and more signal processing based tools are adopted to these problems.

\section{ACKNOWLEDGMENTS}

This work is supported by EC within FP7 under Grant 216105 with the acronym Real 3D.

\section{REFERENCES}

[1] Onural, L., "Television in 3D: What are the prospects," Proceedings of the IEEE 95(6), 1143-1145 (2007).

[2] Darrah, W. C., [The World of Stereographs] (1977).

[3] Holliman, N. S., "Three-dimensional display systems," in [Handbook of Optoelectronics, vol.II], Dakin, J. P. and Brown, R. G. W., eds., Taylor and Francis (2006).

[4] Hale, K. S. and Stanney, K. M., "Effects of low stereo acuity on performance, presence and sickness within a virtual environment," Appl. Ergonomics 37(3), 329 - 339 (2006).

[5] Takada, H., Fujikake, K., and Miyao, M., "On a qualitative method to evaluate motion sickness induced by stereoscopic images on liquid crystal displays," VMR '09: Proceedings of the 3rd International Conference on Virtual and Mixed Reality, 254-262, Springer-Verlag (2009).

[6] Arimoto, H. and Javidi, B., "Integral three-dimensional imaging with digital reconstruction," Opt. Lett. 26(3), 157-159 (2001).

[7] Jang, J.-S. and Javidi, B., "Improved viewing resolution of three-dimensional integral imaging by use of nonstationary micro-optics," Opt. Lett. 27(5), 324-326 (2002).

[8] Frauel, Y. and Javidi, B., "Digital three-dimensional image correlation by use of computer-reconstructed integral imaging," Appl. Opt. 41(26), 5488-5496 (2002).

[9] Hariharan, P., [Optical Holography: Principles, Techniques and Applications], Cambridge University Press (1996).

[10] Yaroslavsky, L. P., [Digital Holography and Digital Image Processing], Kluwer Academic, Boston, Mass. (2004).

[11] Sahin, E. and Onural, L., "A comparative study of light field representation and integral imaging," The Imaging Science Journal. In print.

[12] Kovachev, M., Ilieva, R., Benzie, P., Esmer, G. B., Onural, L., Watson, J., and Reyhan, T., "Holographic displays using spatial light modulators," in [Three-Dimensional Television: Capture, Transmission, and Display], Ozaktas, H. M. and Onural, L., eds., ch. 15, Springer Verlag (2007).

[13] Kovachev, M., Ilieva, R., Onural, L., Esmer, G. B., Reyhan, T., Benzie, P., Watson, J., and Mitev, E., "Reconstruction of computer generated holograms by spatial light modulators," in [MRCS], 706-713 (2006).

[14] Reichelt, S., Haussler, R., Leister, N., Futterer, G., and Schwerdtner, A., "Large holographic 3D displays for tomorrows TV and monitors - solutions, challenges, and prospects," IEEE Lasers and Electro-Optics Society, 2008. LEOS 2008. 21st Annual Meeting of the, 194-195 (2008).

[15] Yaraş, F., Kang, H., and Onural, L., "Real-time phase-only color holographic video display system using led illumination," Appl. Opt. 48(34), H48-H53 (2009).

[16] Shimobaba, T., Shiraki, A., Masuda, N., and Ito, T., "An electroholographic colour reconstruction by time division switching of reference lights," J. Opt. A: Pure Appl. Opt. 9, 757-760 (2007). 
[17] U.Schnars, Kreis, T. M., and Jüptner, W. P. O., "Digital recording and numerical reconstruction of holograms: reduction of the spatial frequency spectrum," Optical Engineering 35(4), 977-982 (1996).

[18] Kelly, D. P., Monaghan, D., Pandey, N., Kozacki, T., Michalkiewicz, A., Hennelly, B., Kujawinska, M., and Finke, G., "Digital holographic capture and opto-electronic reconstruction for 3D displays." International Journal of Digital Multimedia Broadcasting. In print.

[19] Onural, L., Gotchev, A., Ozaktas, H. M., and Stoykova, E., "A survey of signal processing problems and tools in holographic three-dimensional television," Circuits and Systems for Video Technology, IEEE Transactions on 17(11), 1631-1646 (2007).

[20] Lucente, M., Diffraction-specific fringe computation for electro-holography, PhD thesis, Cambridge, MA, USA (1995).

[21] Lucente, M., "Optimization of hologram computation for real-time display," in [Practical Holography VI], Benton, S. A., ed., Proc. SPIE 1667, 32-43 (1992).

[22] Kang, H., "Quality improvements of the coherent holographic stereogram for natural 3D display and its applications," in [PhD. Thesis, Nihon University], (2008).

[23] Kang, H., Fujii, T., Yamaguchi, T., and Yoshikawa, H., "Compensated phase-added stereogram for real-time holographic display," Opt. Eng. 46(9), 095802 (2007).

[24] Kang, H., Yaraş, F., and Onural, L., "Graphics processing unit accelerated computation of digital holograms," Appl. Opt. 48(34), H137-H143 (2009).

[25] Yaraş, F., Kang, H., and Onural, L., "Real-time multiple SLM color holographic display using multiple GPU acceleration," in [Digital Holography and Three-Dimensional Imaging], DWA4, Optical Society of America (2009).

[26] Ito, T., Masuda, N., Yoshimura, K., Shiraki, A., Shimobaba, T., and Sugie, T., "Special-purpose computer HORN-5 for a real-time electroholography," Opt. Express 13(6), 1923-1932 (2005).

[27] Ichihashi, Y., Nakayama, H., Ito, T., Masuda, N., T.Shimobaba, Shiraki, A., and Sugie, T., "HORN6 special-purpose clustered computing system for electroholography," Opt. Express 17(16), 13895-13903 (2009).

[28] Janda, M., Hanak, I., and Onural, L., "Hologram synthesis for photorealistic reconstruction," JOSA A 25(12), 3083-3096 (2008).

[29] Ahrenberg, L., Page, A. J., Hennelly, B. M., McDonald, J. B., and Naughton, T. J., "Using commodity graphics hardware for real-time digital hologram view resonstruction," J. of Display Technol. 5(4), 111-119 (2009).

[30] Ahrenberg, L., Benzie, P., Magnor, M., and Watson, J., "Computer generated holography using parallel commodity graphics hardware," Optics Express 14(17), 7656-7641 (2006).

[31] Michalkiewicz, A., Kujawinska, M., Kozackia, T., Wangb, X., and Bosb, P. J., "Holographic threedimensional displays with liquid crystal on silicon spatial light modulator," in [Interferometry XII: Techniques and Analysis], Proc. of SPIE, 85-94 (2004).

[32] Ito, T., Shimobaba, T., Godo, H., and Horiuchi, M., "Holographic reconstruction with a $10-\mu m$ pixelpitch reflective liquid-crystal display by use of a light-emitting diode reference light," Optics Letters 27(16), 1406-1408 (2002).

[33] Yaras, F., Kovachev, M., Ilieva, R., Agour, M., and Onural, L., "Holographic reconstructions using phaseonly spatial light modulators," in [3DTV Conference: The True Vision - Capture, Transmission and Display of 3D Video, IEEE], PD-1-PD-4 (2008).

[34] Yaras, F. and Onural, L., "Color holographic reconstruction using multiple SLMs and LED illumination," in [Proc. SPIE.], 7237, 723700 (2009).

[35] Sato, K., Sugita, A., Morimoto, M., and Fujii, K., "Reconstruction of full-color images with a RGB timesharing holographic display system," in [Display Devices and Systems], Proc. of SPIE 6030, 603004-1603004-6 (2006).

[36] Sato, K., Sugita, A., Morimoto, M., and Fujii, K., "Reconstruction of color images of high quality by a holographic display," in [Practical Holography XX: Materials and Applications], Proc. of SPIE 6136, 61360V-1-61360V-9 (2006). 
[37] Sugita, A., Sato, K., Morimoto, M., and Fujii, K., "Full-color holographic display and recording of 3D images," in [Practical Holography XIX: Materials and Applications], Proc. of SPIE 5742, 130-139 (2005).

[38] Shimobaba, T. and Ito, T., "A color holographic reconstruction system by time division multiplexing with reference lights of laser," Opt. Rev. 10(5), 339-341 (2009).

[39] Ito, T. and Okano, K., "Color electroholography by three colored reference lights simultaneously incident upon one hologram panel," Opt. Express. 12(18), 4320-4325 (2004).

[40] Takano, K., Minami, N., and Sato, K., "A simple method of color electro-holographic display system using a white light source and three LCD panels," in [IEEE International Conference on Multimedia and Expo], 105-108 (2001).

[41] Takano, K. and Sato, K., "Color electro-holographic display using a single white light source and a focal adjustment method," Opt. Eng. 41(10), 2427-2433 (2002).

[42] Takano, K., Minami, N., and Sato, K., "Practical method for color electro-holographic display system using a metal halide lamp and three LCD panels," in [Practical Holography XVI and Holographic Materials VIII], Proc. of SPIE 4659, 61-68 (2002).

[43] Sato, K. and Takano, K., "New type electro-holographic display system using LCDs," in [Three-Dimensional TV, Video, and Display], Proc. of SPIE 4864, 114-121 (2002).

[44] Takano, K. and Sato, K., "Full-color electroholographic three-dimensional display system employing light emitting diodes in virtual image reconstruction," Opt. Eng. 46(9), 095801 (2007).

[45] Sato, K. and Takano, K., "Consideration about HMD-type holography 3D-TV," in [Three-Dimensional TV, Video, and Display III], Proc. of SPIE 5599, 123-134 (2004).

[46] Hahn, J., Kim, H., Lim, Y., Park, G., and Lee, B., "Wide viewing angle dynamic holographic stereogram with a curved array of spatial light modulators," Opt. Express 16(16), 12372-12386 (2008).

[47] Hilaire, P. S., Benton, S. A., Lucente, M., and Hubel, P. M., "Color images with the MIT holographic video display," in [Practical Holography VI], Benton, S. A., ed., Proc. of SPIE 1667, 73-84 (1992).

[48] St.-Hilaire, P., "Scalable optical architectures for electronic holography," in [Ph. D. Thesis, Program in Media Arts and Sciences, MIT], (1994).

[49] Hilaire, P. S., Benton, S. A., Lucente, M., Sutter, J. D., , and Plesniak, W. J., "Advances in holographic video," in [Practical Holography VII], Proc. of SPIE (1993).

[50] Hillaire, P. S., Lucente, M., Sutter, J., Pappu, R., C.J.Sparrell, , and Benton, S., "Scaling up the MIT holographic video system," in [Proc. of the Fifth Inter. Symposium on Display Holography], (1995).

[51] Jepsen, M. L., "Holographic video: design and implementation of a display system," in [M.S. Thesis, Department of Architecture, MIT], (1989).

[52] Kollin, J. S., "Design and information considerations for holographic television," in [M.S. Thesis, Department of Architecture, MIT], (1988).

[53] Lucente, M. and Galyean, T., "Rendering interactive holographic images," in [Proc. of SIGGRAPH '95], 387-394 (1995).

[54] Underkoffler, J., "Towards accurate computation of optically reconstructed holograms," in [M.S. Thesis, Program in Media Arts and Sciences, MIT], (1991).

[55] Häussler, R., Schwerdtner, A., and Leister, N., "Large holographic displays as an alternative to stereoscopic displays," Stereoscopic Displays and Applications XIX 6803(1), 68030M, SPIE (2008).

[56] Reichelt, S., Sahm, H., Leister, N., and Schwerdtner, A., "Capabilities of diffractive optical elements for real-time holographic displays," in [Practical Holography XXII: Materials and Applications], Proc. of SPIE 6912(1), 69120P (2008).

[57] Schwerdtner, A., Häussler, R., and Leister, N., "Large holographic displays for real-time applications," in [Practical Holography XXII: Materials and Applications], SPIE 6912(1), 69120T (2008).

[58] Leister, N., Schwerdtner, A., G.Fütterer, Buschbeck, S., Olaya, J.-C., and Flon, S., "Full-color interactive holographic projection system for large 3D scene reconstruction," Emerging Liquid Crystal Technologies III 6911(1), 69110V, SPIE (2008).

[59] Stanley, M., Smith, M. A. G., Smith, A. P., Watson, P. J., Coomber, S. D., Cameron, C. D., Slinger, C. W., and Wood, A. D., "3D electronic holography display system using a 100 mega-pixel spatial light modulator," in [Optical Design and Engineering], Proc. of SPIE 5249, 297-308 (2004). 
[60] Shimobaba, T., Hishinuma, S., and Ito, T., "Special-purpose computer for holography HORN-4 with recurrence algorithm," Computer Physics Communications 148(2), 160 - 170 (2002).

[61] Yabe, T., Ito, T., and Okazaki, M., "Holography machine HORN-1 for computer-aided retrieval of virtual three-dimensional image," Japanese journal of applied physics. Pt. 2, Letters 32(9), L1359-L1361 (1993).

[62] Ichihashi, Y., Ito, T., Nakayama, H., Masuda, N., Shiraki, A., and Shimobaba, T., "Development of specialpurpose computer HORN-6 for holography," 3D Image Conference 2008 (2008).

[63] Shimobaba, T., Masuda, N., Sugie, T., Hosono, S., Tsukui, S., and Ito, T., "Special-purpose computer for holography HORN-3 with PLD technology," Computer Physics Communications 130 (July 2000).

[64] Ito, T., Yabe, T., Okazaki, M., and Yanagi, M., "Special-purpose computer HORN-1 for reconstruction of virtual image in three dimensions," Comp. Phys. Commun. 82, 104-110 (1994).

[65] Ito, T., Eldeib, H., Yoshida, K., Takahashi, S., Yabe, T., and Kunugi, T., "Special-purpose computer for holography HORN-2," Comp. Phys. Commun. 93, 13-20 (1996).

[66] "http://www.televisionbroadcast.com/article/79134."

[67] Watlington, J. A., Lucente, M. E., Sparrell, C. J., V. M. Bove, J., and Tamitani, I., "Hardware architecture for rapid generation of electro-holographic fringe patterns," in [Practical Holography IX], Benton, S. A., ed., Proc. SPIE 2406(1), 172-183 (1995).

[68] Okada, T., Iwata, S., Nishikawa, O., Matsumoto, K., Yoshikawa, H., Sato, K., and Honda, T., "Fast computation of holograms for the interactive holographic 3D display system," International Conference on Applications of Optical Holography 2577(1), 33-40, SPIE (1995).

[69] Lucente, M., "Interactive computation of holograms using a look-up table," Journal of Electronic Imaging $\mathbf{2}$, 28-34 (1993).

[70] Yoshikawa, H., Iwase, S., and Oneda, T., "Fast computation of fresnel holograms employing difference," Practical Holography XIV and Holographic Materials VI 3956(1), 48-55 (2000).

[71] Kang, H., Yamaguchi, T., and Yoshikawa, H., "Accurate phase-added stereogram," in [Adaptive Optics: Analysis and Methods/Computational Optical Sensing and Imaging/Information Photonics/Signal Recovery and Synthesis Topical Meetings on CD-ROM], DTuB5, Optical Society of America (2007).

[72] Kang, H., Yamaguchi, T., and Yoshikawa, H., "GPU-based acceleration method for coherent holographic stereogram calculation," in [Digital Holography and Three-Dimensional Imaging], DWA4, Optical Society of America (2008).

[73] Kang, H., Yamaguchi, T., Yoshikawa, H., Kim, S.-C., and Kim, E.-S., "Acceleration method of computing a compensated phase-added stereogram on a graphic processing unit," Appl. Opt. 47(31), 5784-5789 (2008).

[74] Kang, H., Yaraş, F., Onural, L., and Yoshikawa, H., "Real-time fringe pattern generation with high quality," in [Digital Holography and Three-Dimensional Imaging], DTuB7, Optical Society of America (2009).

[75] Mills, G. A. and Yamaguchi, I., "Effects of quantization in phase-shifting digital holography," Appl. Opt. 44(7), 1216-1225 (2005). 